\title{
A relação terapêutica na Terapia Cognitivo-Comportamental
}

\section{The therapeutic relationship in Cognitive-Behavioral Therapy}

Camille Bueno ${ }^{1 *}$, Andréia Cristina dos Santos Kleinhans ${ }^{2}$, Diego da Silva ${ }^{1}$,

\section{RESUMO}

O presente trabalho tem por objetivo refletir sobre a importância da relação terapêutica na Terapia Cognitivo-Comportamental. Para tanto foi realizada pesquisa de revisão narrativa de literatura em bases de dados científicos como Pepsic, Scielo, Biblioteca Virtual do Grupo Uninter, Google Acadêmico, Bireme, Pubmed, livros da Terapia Cognitivo-Comportamental e sites oficiais que versem sobre a temática em questão. A Relação Terapêutica é essencial para que ocorra alguma mudança na vida do paciente. A psicoterapia se dá num contexto de relação interpessoal, portanto, o paciente precisa estabelecer um bom vínculo com o terapeuta, sendo que isto só será possível se houver empatia, capacidade para escuta qualificada e uma boa habilidade para realizar orientações/ encaminhamentos.

Palavras-chave: Relação Terapêutica; Terapia Cognitivo-Comportamental; Psicologia.

\section{ABSTRACT}

The present work aims to reflect on the importance of the therapeutic relationship in Cognitive-Behavioral Therapy. For this purpose, a narrative literature review research was carried out in scientific databases such as Pepsic, Scielo, Uninter Group Virtual Library, Academic Google, Bireme, Pubmed, CognitiveBehavioral Therapy books and official websites dealing with the subject in question. The Therapeutic Relationship is essential for any change to occur in the patient's life. Psychotherapy takes place in an interpersonal relationship context, therefore, the patient needs to establish a good bond with the therapist, and this will only be possible if there is empathy, qualified listening skills and a good ability to carry out orientations/referrals.

Keywords: Therapeutic Relationship; Cognitive behavioral therapy; Psychology.

\footnotetext{
${ }^{1}$ Especialização em Terapia Cognitivo- Comportamental pelo Grupo UNINTER, Curitiba, Paraná.

*E-mail: psicamillebueno@gmail.com

${ }^{2}$ Curso de Psicologia, UniEnsino, Curitiba, Paraná.
} 


\section{INTRODUÇÃO}

A relação terapêutica é um dos temas que precisa ser discutido dentro do trabalho dos psicólogos, haja vista que este fator será preponderante para o sucesso da psicoterapia, que neste artigo, tem o foco da psicoterapia cognitivo-comportamental. Sendo assim, vale entender o que seria a Terapia Cognitivo-Comportamental (TCC). Para Sousa e Padovani (2015) terapeutas cognitivo-comportamentais realizam intervenções para reduzir sofrimento a partir da compreensão da subjetividade humana, entendida como uma interação recíproca entre crenças, emoções, comportamentos, fisiologia e ambiente. Portanto, os terapeutas devem ser treinados para entender a relação entre as crenças, as emoções, os comportamentos, a fisiologia, o ambiente e os problemas apresentados pelo paciente, isto é, desenvolver a habilidade de fazer a conceitualização de pacientes que sejam atendidos.

De acordo com Serra (2020) a TCC reflete aspectos interessantes em sua prática clínica. Baseia-se na noção de esquemas, construídos ao longo do desenvolvimento, cujo conjunto resume as percepções pelo indivíduo de regularidades do real com base em suas experiências históricas relevantes. Isto a partir de estruturas cognitivas que, em uma relação multimodal, organizam nossas experiências do real e são atualizadas por elas, ao mesmo tempo em que guiam o foco de nossa atenção. A TCC adota uma abordagem estruturada, mas apoia-se em uma relação bilateral entre o terapeuta e o cliente, na qual ambos têm um papel ativo através do processo psicoterápico. Objetiva não apenas a resolução dos problemas imediatos do indivíduo, mas, através da reestruturação cognitiva, busca mostrar a ele um novo conjunto de técnicas e estratégias cognitivas para, a partir daí, processar e responder ao real de forma funcional.

A relação terapêutica irá fazer parte de todo esse processo, portanto, é necessário caracterizá-la. Segundo Prado e Meyer (2004) a relação terapêutica poderá exercer influência positiva, se o terapeuta tiver participação efetiva no tratamento, já que, tendose desenvolvido uma relação terapêutica positiva, o cliente sente-se confortável para fornecer as informações necessárias para a terapia, confiando no terapeuta suas informações mais sigilosas e difíceis de falar e lidar. Deste modo, a relação terapêutica pode ser vista como fator determinante do processo terapêutico, a qual pode facilitar o trabalho e a possibilidade de atingir metas, caso se estabeleça num clima de confiança e acordo harmonioso. 
Prado e Meyer (2004) colocam ainda que três fatores são preponderantes para que a relação terapêutica seja potencializada. A empatia, a escuta qualificada e a capacidade de orientar (Psicoeducação) os pacientes, isto obviamente dentro de uma abordagem Cognitivo-Comportamental. Sampaio, Camino e Roazzi (2019) apontam que a empatia é vista como um construto que reflete respostas afetivas e cognitivas ligadas a situações específicas, sendo, portanto, necessária em qualquer processo terapêutico para que se obtenha sucesso no mesmo. Uma proposição básica dessa perspectiva é a de que o nível de empatia varia de momento para momento, e que o estudo de fatores situacionais é importante para poder entendê-la. O terapeuta deve focalizar a atenção nos estímulos que se fazem mais salientes no momento em que a empatia é produzida. Tendo empatia, a escuta deste terapeuta será mais efetiva e atenciosa, o que consequentemente irá gerar mais segurança nele para realizar orientações adequadas aos mesmos.

O estudo do presente tema é relevante para o meio acadêmico e para a comunidade científica da TCC, no sentido de que beneficia e promove intervenções clínicas reguladas em condições teórico/práticas, especialmente aos recém-formados na graduação. Desta forma, o terapeuta pode ter bases concretas em seus atendimentos profissionais e os clientes terão a probabilidade de culminante sucesso no processo terapêutico. A profissão do psicólogo ainda é pouco difundida entre a população gerando uma série de mitos e receios, sendo assim, o presente estudo pode permitir que os clientes entendam melhor a Psicologia.

A presente pesquisa é de cunho bibliográfico e qualitativo, sendo do tipo revisão narrativa de literatura. As fontes de pesquisa serão as bases de dados científicos como Pepsic, Scielo, Biblioteca Virtual do Grupo Uninter, Google Acadêmico, Bireme, Pubmed, livros da Terapia Cognitivo-Comportamental e sites oficiais que versem sobre a temática em questão. Os trabalhos selecionados para pesquisa deverão ter sido publicados nos últimos 15 anos sendo que os descritores de busca englobaram "Relação Terapêutica e Terapia Cognitivo-Comportamental"; "Psicologia e Terapia CognitivoComportamental". Os idiomas selecionados foram português, inglês e espanhol, tendo como critérios de seleção manuscritos que continham bases teóricas e práticas sólidas sobre o assunto deste artigo.

O presente artigo tem por objetivo verificar a importância da relação terapêutica no processo da terapia cognitivo-comportamental. Para tanto será necessário discorrer sobre a relação terapêutica no que tange ao seu conceito, mas, além disso, será necessário 
entender quais comportamentos do terapeuta são considerados primordiais para que o sucesso da psicoterapia seja possível e para que o paciente consiga dar continuidade em suas mudanças de comportamento.

\section{TERAPIA COGNITIVO-COMPORTAMENTAL}

A Terapia Cognitivo Comportamental ou TCC é uma abordagem da psicoterapia que tem como origem o Behaviorismo e teorias cognitivas. Esta forma de tratamento procura entender como a pessoa interpreta os acontecimentos e como as emoções acontecem a partir dos pensamentos, e não os acontecimentos em si. Ou seja, é a forma como cada um vê, sente e pensa com relação à uma situação que causa desconforto, dor, incômodo, tristeza ou qualquer outra sensação. A Terapia Cognitiva foi fundada no início dos anos 60 por Aaron Beck, Neurologista e Psiquiatra norte-americano. Beck propôs, inicialmente, um "modelo cognitivo da depressão" e que posteriormente evoluiu para a compreensão e tratamento de outros transtornos. (NOGUEIRA, 2018).

$\mathrm{Na}$ década de 80, foi desenvolvido um modelo de intervenção cognitivocomportamental para pessoas com impulsividade. Ele objetiva desenvolver estratégias de automanejo dos sintomas relacionados com a impulsividade e procura capacitar o indivíduo para automonitorizar o seu comportamento, identificar e antecipar situações problemáticas e empregar uma estratégia de solução de problemas, mediada por autoinstruções para resolvê-los (HOUNIE E CAMARGOS, 2005).

Uma das abordagens psicoterapêuticas mais utilizadas em intervenções clínicas é a Terapia Cognitivo-Comportamental. A TCC é um termo genérico que abrange mais de 20 abordagens de psicoterapias derivadas do modelo cognitivo e cognitivo-comportamental. Ela tem encontrado evidências de eficácia e efetividade para o tratamento de diversos transtornos mentais e caracteriza-se como uma psicoterapia breve, com o foco no presente, estruturada, direcionada para resolver problemas atuais e modificar os comportamentos e pensamentos disfuncionais. Várias abordagens derivadas da TCC emergiram ao longo dos anos desde o seu surgimento, mas todas assumem três pressupostos básicos comuns: o primeiro diz respeito ao papel mediacional da cognição, em que propõe que há sempre processamento cognitivo que pode influenciar respostas a eventos internos e externos; a segunda afirma que a atividade cognitiva pode ser avaliada, monitorada e medida; e a terceira defende que o comportamento almejado pode ser influenciado por meio da mudança cognitiva (SINGULANE e SARTE, 2017, p. 785). 
Essa abordagem é bastante específica, clara e direta. É utilizada para tratar diversos transtornos mentais de forma eficiente. Seu objetivo principal é identificar padrões de comportamento, pensamento, crenças e hábitos que estão na origem dos problemas, indicando, técnicas para alterar essas percepções de forma positiva. A TCC se destina tanto ao tratamento dos diferentes transtornos psicológicos e emocionais quanto para outras situações de busca da psicoterapia (NOGUEIRA, 2018).

O Psicoterapeuta identifica os padrões e as crenças que são construídas pelos pacientes gerando pensamentos e emoções que refletem nos comportamentos. Diante dos padrões mal adaptativos ou disfuncionais de pensamentos, cabe ao terapeuta auxiliar o paciente a encontrar novas possibilidades de pensamentos alternativos e mais funcionais que possibilitem uma boa adaptação à sua realidade social. Isso é feito a partir da determinação de um foco e de metas para que, com o tempo, o paciente adquira sua autonomia e possa lidar com as questões de forma independente. Esta é a reestruturação cognitiva e comportamental que dá nome à abordagem (BECK et al., 1987).

As intervenções cognitivas enfatizam o fato de que os comportamentos derivam do modo como os indivíduos percebem e representam a sua situação, procurando promover a auto-observação e o insight (HOUNIE e CAMARGOS, 2005). Tudo isto só é possível se houver uma relação terapêutica adequada entre terapeuta e cliente, deste modo, o próximo tópico irá discorrer e refletir sobre a importância da Relação Terapêutica.

\section{RELAÇÃO TERAPÊUTICA}

A Relação Terapêutica é essencial para que ocorra alguma mudança na vida do paciente. A psicoterapia se dá num contexto de relação interpessoal. O paciente precisa estabelecer um bom vínculo com o terapeuta. Para que esse vínculo seja estabelecido o terapeuta deve ter como características pessoais empatia, calor humano, interesse em falar com as pessoas e ouví-las, etc (CORDIOLI et al., 2008).

Para a abordagem cognitivo-comportamental o estabelecimento de uma aliança entre terapeuta e cliente é fundamental, pois propicia um ambiente de segurança e confiança, condições necessárias para aprender, implementar e praticar as técnicas trabalhadas em psicoterapia. A aliança terapêutica ou aliança terapêutica de trabalho é um dos componentes que constituem a relação terapêutica. A aliança 
terapêutica é uma relação de colaboração mútua, marcada por concordâncias entre terapeuta e cliente e constituída por três componentes interdependentes: objetivos, tarefas e vínculo. Os objetivos dizem respeito ao consenso sobre expectativas de resultados de curto e longo prazo entre terapeuta e cliente. As tarefas podem ser definidas como acordos ou consensos entre terapeuta e cliente no que diz respeito ao que deve ser feito na terapia e como atividades diversas na terapia contribuirão para a resolução do problema do cliente. Já o vínculo está associado à ligação afetiva entre terapeuta e cliente (SINGULANE e SARTE, 2017, p. 786).

Del Prette, Del Prette e Meyer (2007) fizeram um levantamento das expectativas e habilidades sociais de graduandos em Psicologia com relação a psicoterapia e a relação terapêutica. Chegaram à conclusão que diferentes habilidades terapêuticas são necessárias ao psicólogo clínico para uma melhor relação com os clientes, como acolher, estimular, escutar, compreender, ser empático, assertividade, a expressividade emocional e a habilidade de perceber aspectos de si durante o atendimento, descrição em termos de autosensação, autopercepção, auto-observação e autoconhecimento, usar o nome do cliente ao se dirigir a ele, manter contato visual, adaptar sua linguagem à do cliente, entre outras. Da mesma forma, Del Prette et. al (2004) acrescentam qualidades como fazer e responder cumprimentos e elogios, expressar opiniões e discordâncias, iniciar, manter e encerrar conversações, fazer críticas e responder a elas, tom de voz, contato visual, gestos, postura, pensamentos, percepções, representações, entre outros.

Zaluf (2006) realizou uma pesquisa para identificar aspectos que influenciam no sucesso de estudantes do ensino superior para sua empregabilidade, ou seja, para que possuam sucesso em suas carreiras futuras, em qualquer área de formação. A relação terapêutica positiva com os clientes foi a primeira premissa encontrada nos resultados da pesquisa. Para isso, alguns aspectos foram apontados, como por exemplo a comunicação, habilidades matemáticas, tecnologia de informação, habilidades pessoais e interpessoais, capacidade de solucionar problemas, autoconfiança, aprendizagem, autoconhecimento, autopromoção, saber aproveitar e criar oportunidades, planejamento de ação, saber trabalhar em rede, habilidades de conciliação, de tomada de decisão, de negociação, ter consciência política, saber lidar com a incerteza, enfocar o desenvolvimento, habilidades de transferir conhecimentos adquiridos para diversas situações, conhecimento, intelecto, entusiasmo e a perseverança, avaliação crítica, transferência da teoria à prática, flexibilidade, entre outras. As habilidades de empregabilidade e relação terapêutica incluem competências gerais, pessoais e intelectuais. 
Para Bartholomeu, Nunes e Machado (2008) o comportamento socialmente competente e que irá influenciar na relação terapêutica é definido como aquele que as pessoas consideram apropriado em uma situação específica. Nesse contexto, o que determina uma resposta socialmente hábil é a sua eficácia ou consequência numa dada situação. Socialização é um importante componente da personalidade humana. As tendências apontadas para pessoas com comportamento socialmente competente são agradabilidade social, amabilidade, altruísmo, cuidado com os demais, amor e apoio emocional. São normalmente generosas, bondosas, afáveis, prestativas e altruístas. Ávidas para ajudar aos outros, apresentam ainda tendência a ser responsivas e empáticas, e acreditam que a maioria das outras pessoas irá agir da mesma forma.

Por sua vez, pessoas com baixos escores em socialização seriam descritas como cínicas, não-cooperativas e irritáveis, podendo também ser manipuladoras, vingativas e implacáveis. Caracterizam-se ainda por hostilidade, indiferença aos demais, egoísmo e inveja. Estes podem ser considerados alguns dos itens que beneficiam ou prejudicam a psicoterapia se fazem parte da vida e da personalidade dos terapeutas (BARTHOLOMEU et al, 2008). Couto, Vandenbergue, Tavares e Silva (2012) colocam que pessoas com melhores habilidades sociais apresentam padrões de interação mais adequados. Por exemplo, no grupo de altas habilidades que se notou a presença de maior variedade de padrões interpessoais e atitudes mais ativas, como a demonstração de interesse pelo ambiente social, busca pelo contato e crença na própria capacidade de contribuir para as decisões sobre os caminhos que o grupo deve seguir.

No grupo com habilidades sociais mais restritas, as pessoas apresentavam mais posições interpessoais passivas, como retraimento social, sentimento de inadequação, falta de interesse em assumir responsabilidades sociais etc. Posições interpessoais específicas, socialmente indesejáveis, mostraram-se mais relacionadas com a falta de habilidade social, enquanto posições interpessoais desejáveis mostraram-se relacionadas com a presença dessas habilidades. Sugere-se que atitudes problemáticas específicas, quando aparecem no grupo, prejudicam sua competência social. Por outro lado, um desempenho socialmente hábil não é garantido apenas pela presença de determinadas posições interpessoais, por exemplo, a simples demonstração de cordialidade, deferência ou aceitação do outro. A pessoa deve mesclar tipos de atitudes amigáveis, ora ativas, ora passivas, e principalmente, sobrepor as atitudes amigáveis às hostis (COUTO et al, 2012). 


\section{A RELAÇÃO TERAPÊUTICA NA TERAPIA COGNITIVO- COMPORTAMENTAL}

Uma vez que se compreende a importância da relação terapêutica no contexto da Psicologia, faz necessário perceber e refletir como esta relação pode ser desenvolvida e trabalhada no contexto clínico da TCC. Nos tópicos a seguir serão mencionados os três elementos preponderantes (empatia, escuta qualificada e habilidade para psicoeducação) para que a relação terapêutica seja fluída entre cliente e terapeuta, conforme dados de Prado e Meyer (2004).

\section{EMPATIA}

De acordo com Sampaio, Camino e Roazzi (2009) o termo empatia deriva da palavra grega "empatheia". Este termo era utilizado dentro d apalavra grega no ambiente das artes, como um processo de imitação interna chamado de Einfühlung, ocorrido durante a apreciação de objetos de arte e no qual a projeção do self em obras artísticas fazia com que sentimentos de admiração e unicidade surgissem nos observadores dessas obras. Através do Einfühlung, propriedades subjetivas como nobreza, elegância e poder podiam ser sentidas como se pertencessem às próprias obras de arte, tal como se estas fossem seres com vida. Obviamente, isto ocorria no tempo da idade média quando se iniciou a apreciação das artes e do processo civilizatório.

Para Miguel et al (2018) empatia é definida como a capacidade de compreender os estados emocionais das outras pessoas e de vivenciar suas mesmas emoções, manifestando essa capacidade de forma que as outras pessoas se sintam compreendidas. A empatia pode ser dividida em dois processos: cognitivo e afetivo. A empatia cognitiva implica compreender racionalmente o que as outras pessoas estão sentindo, seus motivos e propósitos, sem necessariamente experimentar seus mesmos sentimentos.

Já a empatia afetiva, também chamada de contágio emocional, diz respeito a experimentar as mesmas emoções que as outras pessoas estão vivenciando, como sentirse triste quando outra pessoa está triste. Mais recentemente identifica-se um terceiro componente da empatia, chamado de pró-sociabilidade ou compaixão, indicando desejo de ajudar e evitar que eventos desagradáveis aconteçam às outras pessoas. Pesquisas têm demonstrado que a empatia está relacionada a diversos aspectos psicológicos, como traços de personalidade. Nesse sentido, costuma estar predominantemente associada ao 
traço agradabilidade, indicando estar relacionada a maior preocupação com a qualidade das interações sociais e pró-sociabilidade (MIGUEL et al., 2018).

Segundo Sampaio et al (2009) a empatia está diretamente relacionada ao desenvolvimento de um senso cognitivo sobre a existência de outras pessoas, o qual, por sua vez, se encontra ligado ao processo de diferenciação do self. Além disso, a associação entre os sentimentos produzidos durante os episódios empáticos e os níveis de desenvolvimento sociocognitivo produz mudanças na maneira como os indivíduos irão sentir subjetivamente a empatia. Com o desenvolvimento do sentido cognitivo do outro e com a transferência de parte dos afetos empaticamente sentidos no self para a imagem do outro, a angústia empática pode ser parcialmente transformada em um novo tipo de sentimento, chamado de angústia simpática. Por meio desse sentimento, o sujeito experiencia um sentimento de piedade ou compaixão pelo paciente e sente necessidade não só de aliviar sua própria angústia ou sofrimento, mas também um desejo claro de ajudar o outro.

A empatia cumpre um papel social fundamental, tornando possível o compartilhamento de experiências, necessidades e objetivo entre os indivíduos. Isto porque a habilidade de entender as intenções e as crenças do outro é crucial para o sucesso das interações sociais. Nas relações e ambientes da saúde, a empatia é definida como um atributo com dimensões emocionais e cognitivas que possibilita uma compreensão das experiências interiores e da perspectiva do paciente como um indivíduo singular, somada à capacidade de comunicar esse entendimento ao paciente. A profundidade da relação terapeuta-paciente está vinculada a quatro elementos principais: conhecimento, confiança, lealdade e respeito. A função da empatia seria identificar e compreender os sentimentos do paciente e tomar da sua perspectiva, promovendo, assim, um aumento na confiança, na lealdade e no respeito entre ambos. Um aumento na empatia leva a um incremento na satisfação dos pacientes com o atendimento, além de maior adesão ao tratamento proposto (SUARTZ, 2013).

\section{ESCUTA QUALIFICADA}

Para Rodrigues e Cavalcante (2015) a escuta qualificada permite adquirir informações sobre cada paciente, que poderão mostrar escolhas e resoluções de suas necessidades, tornando-se uma forma de prestar uma assistência de qualidade, pois, por 
meio dela, é possível reconhecer e acolher, empaticamente, as necessidades do paciente, bem como de seus familiares, auxiliando assim na assistência psicológica prestada. No acolhimento deste paciente, durante a primeira entrevista, percebe-se que o paciente indica diversas sensações negativas, por encontrar-se em um ambiente totalmente desconhecido e que ainda, na maioria das vezes, lhe proporciona medo e insegurança. Assim, a escuta qualificada acaba por representar uma estratégia importante para a prática dos cuidados, auxiliando na elaboração de um plano terapêutico.

Segundo Duarte et al (2017) a escuta qualificada, que consiste em operar os processos de trabalho em saúde de forma a atender os que procuram os serviços de saúde, principalmente consultórios de Psicologia, ouvindo seus pedidos e assumindo no serviço uma postura capaz de acolher e pactuar respostas mais adequadas aos clientes, implicando em uma escuta ampliada, em ambientes adequados, respeitando a singularidade deles, considerando os motivos que os levaram a buscar o serviço, identificando suas necessidades e dando encaminhamento para a solução de seus problemas. Portanto, é preciso entender a potencialidade que a Escuta Qualificada proporciona, com o objetivo de identificar as demandas de saúde que estão implícitas no contexto social dos sujeitos, possibilitando intervenções eficazes, contribuindo para a mudança do processo de trabalho em saúde, estabelecendo vínculo/ responsabilidade dos profissionais com os pacientes e aumentando, assim, a capacidade de escuta às demandas apresentadas.

De acordo com Passos et al (2020) em geral, a escuta qualificada é bastante associada ao cuidado em saúde mental. A funcionalidade dela não é formada somente pelo ato de ouvir o cliente, mas também de criar uma integração de confiança na comunicação e na relação profissional-paciente. Também é formada pelo ato de desenvolver um plano de cuidado clínico, que possibilite a minimização de angústias pessoais, seja elas quais forem. A escuta é parte do processo que se realiza nas interações mais humanas da profissão. Experiências pessoais são partilhadas e por isso deve ser tratada como uma estratégia valorosa na relação profissional-cliente.

Além disso, a escuta deve ser desempenhada de forma integral e humanizada, o olhar atento do psicólogo deve enxergar seu cliente como um todo, abrangendo a empatia no momento de ouvir e de se colocar no lugar e nas condições vividas por seus pacientes em terapia. A escuta eficiente e qualificada é a assistência adequada da linguagem verbal e não-verbal durante todo esse tratamento, e em todo local de assistência. A forma como os psicólogos agem em todo esse processo, interfere em como os pacientes reagem ao 
tratamento e ao autocuidado de suas angústias. A forma como a linguagem é utilizada pode afetar negativamente ou positivamente o diálogo profissional-cliente. A linguagem não verbal é tão importante quanto a linguagem verbal (PASSOS et al, 2020).

\title{
PSICOEDUCAÇÃO
}

Segundo Oliveira e Dias (2018) o termo psicoeducação começou a ser utilizado na década de 80, referindo-se à transmissão de informações sobre os transtornos mentais para familiares e pacientes psicóticos. Na década de 90, a psicoeducação passou a ser desenvolvida junto a outros grupos que apresentavam outros transtornos mentais (como transtorno do humor bipolar, transtorno do estresse pós-trauma, etc.). Atualmente, esse termo se refere tanto à oferta de informações relevantes aos pacientes sobre o transtorno (diagnóstico, etiologia, funcionamento), o tratamento e o prognóstico, quanto à busca por esclarecimento de dúvidas e correções de informações distorcidas.

\begin{abstract}
A psicoeducação tem por objetivo ampliar o conhecimento do paciente/familiar sobre seu problema, a fim de aumentar a compreensão da sua condição, auxiliar na tomada de decisões com base em informações confiáveis e promover maior adesão ao tratamento. Ela deve ocorrer de forma didática e em linguagem adequada ao públicoalvo, que pode ser constituído por pacientes, familiares, educadores e profissionais da saúde. A transmissão desses conhecimentos assume diferentes formatos: individual ou grupal, palestras, rodas de conversas, manuais, vídeos ou biblioterapia. Dentre os diversos benefícios da psicoeducação, destacam-se o aumento do conhecimento sobre o transtorno, da motivação para a mudança, da participação no tratamento, da satisfação com o tratamento psicológico, a maior adesão ao mesmo e a redução de recaídas (OLIVEIRA e DIAS, 2018, P. 244).
\end{abstract}

Para Justo e Kalil (2004) o objetivo da psicoeducação é fazer do paciente um colaborador ativo, aliado dos profissionais de saúde envolvidos e, consequentemente, tornar o procedimento terapêutico mais efetivo. Isso é algo mais sofisticado do que a simples transmissão de informações "técnicas", embora existam evidências de que isso, por si, já traga benefícios ao tratamento. A psicoeducação pode ser vista como o estabelecimento de um fluxo de informações de terapeuta para paciente e vice-versa. Assim, além de estarem em campo dados objetivos e genéricos sobre o problema que está sendo tratado no consultório e a importância dos medicamentos, com as vicissitudes de 
seu uso, poderão ser expostos e analisados outros fatores que interferem na evolução do transtorno e aspectos específicos, próprios do paciente e de seu meio.

É desejável que o paciente explicite seu modo de entender sua situação, reconhecendo ou não a condição mórbida e sua extensão, assim como a necessidade de cuidados sistematizados como o emprego de psicofármacos etc. Tudo isso deve ser abertamente discutido. Também é importante que se conheça a estrutura sociofamiliar e que se possa contar com a ajuda de parentes mais próximos; que se criem condições de reconhecimento precoce dos sintomas e se estabeleçam estratégias de controle, além de se identificarem fatores de vulnerabilidade próprios do paciente. A psicoeducação é uma tentativa de implementar, no paciente, familiares e profissionais, recursos para lidar com a doença, através do compartilhamento bidirecional de informações relevantes (JUSTO e KALIL, 2004).

\section{CONSIDERAÇÕES FINAIS}

Considera-se que a relação terapêutica é de extrema importância para o processo de terapia dos indivíduos que a buscam. Isto principalmente no sentido de que o estabelecimento de um vínculo entre terapeuta e cliente fornece subsídios para que os terapeutas tenham acesso as informações do cliente, possibilitando que o mesmo desempenhe com maior eficiência seu trabalho, nos feedbacks para o cliente, no prognóstico e no curso do tratamento. O cliente também é favorecido com a relação terapêutica no sentido de que poderá ter um maior autoconhecimento e que seus comportamentos disfuncionais possam ser modificados, uma vez que estes são partes dos objetivos da psicoterapia. Deste modo, faz-se necessário que o estudo de tal tema seja difundido e ampliado para que a qualidade da psicoterapia seja potencializada e possa colaborar com mais pessoas e profissionais.

\section{REFERÊNCIAS}

BARTHOLOMEU, Daniel; NUNES, Carlos Henrique Sancineto da Silva; MACHADO, Afonso Antonio. Traços de personalidade e habilidades sociais em universitários. PsicoUSF (Impr.), Itatiba, v. 13, n. 1, p. 41-50, June 2008. 
BECK, A. T.; RUSH, A. J.; SHAW, B. E.; EMERY, G. Terapia Cognitiva e Depressão. $1^{\text {a }}$ ed. Estados Unidos: Guilford, 1987.

CORDIOLI, Aristides Volpato; GIGLIO, Larriany. Como Atuam as Psicoterapias: Os Agentes de Mudanças e as Principais Estratégias e Intervenções Psicoterápicas. In. CORDIOLI, Aristides Volpato. Psicoterapias: Abordagens Atuais. Porto Alegre: Artmed, 2008. P. 43-57.

COUTO, Gleiber et al. Interações e habilidades sociais entre universitários: um estudo correlacional. Estud. psicol. (Campinas), Campinas, v. 29, supl. 1, p. $667-$ 677, Dec. 2012.

DEL PRETTE, Zilda Aparecida Pereira et al . Habilidades sociais de estudantes de Psicologia: um estudo multicêntrico. Psicol. Reflex. Crit., Porto Alegre, v. 17, n. 3, p. 341-350, 2004.

DEL PRETTE, Giovana; DEL PRETTE, Zilda Aparecida Pereira; MEYER, Sonia Beatriz. Psicoterapia com crianças ou adultos: expectativas e habilidades sociais de graduandos de psicologia. Estud. psicol. (Campinas), Campinas, v. 24, n. 3, p. 305314, Sept. 2007.

DUARTE, L. P.; MOREIRA, D. J. Contribuição da Escuta Qualificada para a Integralidade na Atenção Primária. Rev. Gestão \& Saúde (Brasília) Vol. 08, n. 03, Set. 2017.

HOUNIE, A. G.; CAMARGOS JR, W. Manual clínico do transtorno de deficit de atenção / hiperatividade tdah. Editora info, 2005.

JUSTO, Luis Pereira; CALIL, Helena Maria. Intervenções psicossociais no transtorno bipolar. Rev. psiquiatr. clín., São Paulo, v. 31, n. 2, p. 91-99, 2004.

MIGUEL, Fabiano Koich et al. Estudos de Validade do Questionário Online de Empatia. Trends Psychol., Ribeirão Preto, v. 26, n. 4, p. 2203-2216, out. 2018.

NOGUEIRA, T. M. B. (2018) Terapia Cognitivo Comportamental: O que é, como funciona e quando devo procurar?. Disponível em:

https://www.vittude.com/blog/terapia-cognitivo-comportamental/ acesso em $13 / 12 / 2020$.

OLIVEIRA, Clarissa Tochetto de; DIAS, Ana Cristina Garcia. Psicoeducação do Transtorno do Déficit de Atenção/Hiperatividade: O Que, Como e Para Quem Informar?. Trends Psychol., Ribeirão Preto, v. 26, n. 1, p. 243-261, mar. 2018.

PASSOS, B. S. A importância da escuta qualificada no cuidado clínico de enfermagem ao paciente oncológico. Revista Enfermagem Atual In Derme, v. 94, n. 32, 2020 e$020075,2020$.

PRADO, Oliver Zancul; MEYER, Sonia Beatriz. Relação terapêutica: a perspectiva comportamental, evidências e o inventário de aliança de trabalho (WAI). Rev. bras. ter. comport. cogn., São Paulo, v. 6, n. 2, p. 201-209, dez. 2004. 
RODRIGUES, H. B.; CAVALCANTE, J. H. V. vivência de escuta qualificada no acolhimento da emergência adulta. S A N A R E, ISSNe:2317-7748, V.14 - Suplemento 1 - COPISP - 2015.

SAMPAIO, Leonardo Rodrigues; CAMINO, Cleonice Pereira dos Santos; ROAZZI, Antonio. Revisão de aspectos conceituais, teóricos e metodológicos da empatia. Psicol. cienc. prof., Brasília, v. 29, n. 2, p. 212-227, 2009.

SERRA, A. M. M. Sobre a Terapia Cognitivo-Comportamental. (2020). Disponível em: http://www.itcbr.com/oque.shtml. Acesso em: 26/02/2021.

SINGULANE, Bianca Aparecida Ribeiro; SARTES, Laisa Marcorela Andreoli. Aliança Terapêutica nas Terapias Cognitivo-comportamentais por Videoconferência: uma Revisão da Literatura. Psicol. cienc. prof., Brasília, v. 37, n. 3, p. 784-798, set. 2017.

SOUSA, Conceição Reis de; PADOVANI, Ricardo da Costa. Supervisão em Terapias Cognitivo-Comportamentais: Trilhando outros Caminhos Além do ServiçoEscola. Psico-USF, Itatiba, v. 20, n. 3, p. 461-470, dez. 2015.

SUARTZ, Caio Vinícius et al . Avaliação de empatia em residentes de especialidades clínicas e cirúrgicas da Universidade Federal de São Paulo. Rev. bras. educ. med., Rio de Janeiro, v. 37, n. 3, p. 320-325, set. 2013.

ZULAUF, Monika. Ensino superior e desenvolvimento de habilidades para a empregabilidade: explorando a visão dos estudantes. Sociologias, Porto Alegre, n. 16, p. 126-155, Dec. 2006.

\section{Recebido em: 05/11/2021}

Aprovado em: 02/12/2021

Publicado em: 08/12/2021 